TITLE:

\title{
First-principles study in Fe grain boundary with Al segregation: variation in electronic structures with straining
}

\author{
AUTHOR(S):
}

Yuasa, Motohiro; Mabuchi, Mamoru

\section{CITATION:}

Yuasa, Motohiro ... [et al]. First-principles study in Fe grain boundary with Al segregation: variation in electronic structures with straining. Philosophical Magazine 2013, 93(6): 635647

ISSUE DATE:

2013-02

URL:

http://hdl.handle.net/2433/187152

\section{RIGHT:}

(C) 2013 Taylor \& Francis; この論文は出版社版でありません。引用の際 には出版社版をご確認ご利用ください。; This is not the published version. Please cite only the published version. 


\section{First-principles study in Fe grain boundary with Al segregation: Variation in electronic structures with straining}

Motohiro Yuasa, and Mamoru Mabuchi*

Department of Energy Science and Technology, Graduate School of Energy

Science, Kyoto University, Yoshidahonmachi, Sakyo-ku, Kyoto 606-8501, Japan

Motohiro Yuasa

Department of Energy Science and Technology, Graduate School of Energy Science, Kyoto University,

Yoshidahonmachi, Sakyo-ku, Kyoto 606-8501, Japan

Tel: +81-75-753-5421; Fax: +81-75-753-5428

E-mail address: yuasamotohiro@t03.mbox.media.kyoto-u.ac.jp

Mamoru Mabuchi (corresponding author)

Department of Energy Science and Technology, Graduate School of Energy Science, Kyoto University,

Yoshidahonmachi, Sakyo-ku, Kyoto 606-8501, Japan

Tel: +81-75-753-5404; Fax: +81-75-753-5428

E-mail address: mabuchi@energy.kyoto-u.ac.jp 


\section{First-principles study in Fe grain boundary with Al segregation: Variation in electronic structures with straining}

First-principles fully relaxed tensile tests were performed on a $\Sigma 3(111) /[1 \overline{1} 0]$ tilt Fe grain boundary (GB) segregated with Al. The effects of Al segregation on bond breaking in the GB were compared with those of $\mathrm{Cu}$ and $\mathrm{P}$, which are typical GB embrittlers because of charge transfer and covalent-like characteristics, respectively. It was suggested by first-principles tensile tests that the intergranular embrittling potency of $\mathrm{Al}$ is as strong as that of $\mathrm{Cu}$. However, this result disagreed with an estimation based on the Rice-Wang thermodynamic model. The first bond breaking site in the Al-segregated GB was the Fe-Fe bond neighbouring the $\mathrm{Al}$ atom, as in the $\mathrm{Cu}$-segregated $\mathrm{GB}$. This is typical of bond breaking due to charge transfer. However, no charge transfer was observed from the $\mathrm{Fe}$ atom to the $\mathrm{Al}$ atom, while the $\mathrm{Fe}$ atom neighbouring the $\mathrm{Al}$ atom showed covalent-like characteristics. It was suggested from investigations of the charge density at the bond critical point that the effect of covalent-like characteristics of $\mathrm{Al}$ on the $\mathrm{Fe}-\mathrm{Fe}$ bond was small in the initial stage of straining, but it increased as the charge density of the Fe-Fe bond decreased with increasing strain. The investigation of the dynamic change in electronic structure also shed light on the difference of bond breaking behaviour in metallic and covalent-like bonding in metallic materials.

Keywords: First-principles calculations; embrittlement; interfacial segregation; grain boundaries

\section{Introduction}

Enhancement of grain boundary (GB) embrittlement by impurity segregation is a critical problem from both industrial and scientific viewpoints, and its mechanism has been discussed for several decades [1-7]. Rice and Wang [1] investigated segregation effects on GB embrittlement from the thermodynamic viewpoint, and showed that the difference in binding energy between the GB and the fracture surface is critical in determining whether an impurity is an embrittler or a cohesion enhancer. Recent 
significant progress in computational technology enables accurate determination of the binding energies by first-principles calculation [6-12]. More recently, first-principles fully relaxed tensile tests have been performed to investigate the variation in the atomic and electronic structures of the GB in relation to strain [13-19]. For example, it has been found by first-principles fully relaxed tensile tests that charge transfer occurs during straining, resulting in a change in mechanical properties [17]. A dynamic change in atomic and electronic structures with straining can give physical insight into the mechanisms for GB embrittlement enhanced by impurity segregation.

A variety of elements have always been added to advanced steels to impart high materials performance. There have been many reports on effects of various elements on Fe GB embrittlement [7,8,10-12,18-22]. Mintz et al. [23] experimentally demonstrated that $\mathrm{Al}$ induces a decrease in ductility of $\mathrm{Fe}$ alloys, while $\mathrm{Al}$ play a vital role in strengthening of Fe alloys. Seah [21] suggested that Al induces GB embrittlement of Fe, on the basis of thermodynamic data and the atomic size. On the other hand, Geng et al. [22] investigated the effects of 42 additional elements on Fe GB decohesion based on first-principles calculations, and showed that $\mathrm{Al}$ is a weak cohesion enhancer, which is different from the systematic trend of other additive elements. Consequently, it is of interest to investigate the decohesion of the Fe GB segregated with Al. In addition, Al exhibits the covalent-like characteristics due to $p$ electrons [24], although it is a metal element. The present authors studied embrittlement in an Fe GB segregated with P and $\mathrm{Cu}[18,19]$. In the P-segregated Fe GB [18], the first atomic bond breaking occurs at an atomic bond between a segregated $\mathrm{P}$ atom and a neighbouring host $\mathrm{Fe}$ atom when the mobility of the bond is reduced because of covalent-like bonding. In contrast, in the $\mathrm{Cu}$ segregated Fe GB [19], it occurs at a bond of host Fe atoms neighbouring a segregated $\mathrm{Cu}$ atom in the case that the bond of the host atoms is weakened because of charge 
transfer. That is, the bonding states exhibit not only metallic but also covalent-like characteristics with segregation, even if in metal such as Fe. As mentioned above, Al has covalent-like characteristics, but its covalent-like characteristics are weaker than those of $\mathrm{P}$. It is also of interest to elucidate the effect of $\mathrm{Al}$ on the bond breaking in the Fe GB.

In the present work, first-principles fully relaxed tensile testing was performed on a $\Sigma 3(111) /[1 \overline{10}]$ tilt $\mathrm{Fe}$ GB segregated with $\mathrm{Al}$, and the effects of $\mathrm{Al}$ on GB decohesion are compared with those of $\mathrm{Cu}$ and $\mathrm{P}$ from the dynamic variation in electronic structures with straining. According to the Rice and Wang thermodynamic $(\mathrm{R}-\mathrm{W})$ model, $\mathrm{Al}$ is a weak cohesion enhancer, while $\mathrm{Cu}$ and $\mathrm{P}$ are strong embrittlers (see Table 1). However, the first-principles fully relaxed tensile test showed that the effect of $\mathrm{Al}$ as an embrittler was as strong as that of $\mathrm{Cu}$ in the present work. The change in electronic structures with straining is critical in Fe GB embrittlement enhanced by $\mathrm{Al}$, and may lead to the nature of bond breaking in metallic and covalent-like bonding.

\section{Computational method}

BCC Fe cells with a $\Sigma 3(111) /[110]$ tilt GB were used: one was a cell without segregation at the GB (the clean GB), the others were cells with $\mathrm{Al}, \mathrm{Cu}$ or $\mathrm{P}$ segregation at the GB (the Al-, Cu-, or P-segregated GBs), in which an Fe atom (Fe2) at the GB was substituted by the segregation atom, as shown in Fig. 1. To estimate if Al has a tendency to segregate into an Fe GB, the segregation energy of $\mathrm{Al}$ was investigated by calculating the total energies in the cases with substitution of an $\mathrm{Al}$ atom for $\mathrm{Fe} 2$ atom (in the $\mathrm{GB}$ ) and with substitution of an $\mathrm{Al}$ atom for $\mathrm{Fe} 4$ atom (in the bulk). The segregation energy of $\mathrm{Al}$ in the $\mathrm{Fe} \mathrm{GB}$ was $-0.56 \mathrm{eV} /$ atom, indicating that the $\mathrm{Al}$-segregated $\mathrm{Fe} \mathrm{GB}$ is energetically stable. Also, the segregation energy of another Al-segregated GB where an 
$\mathrm{Al}$ atom was substituted for the $\mathrm{Fe} 3$ atom was calculated, and its segregation energy was $-0.22 \mathrm{eV} /$ atom. Thus, the substitution for the $\mathrm{Fe} 2$ was energetically favorable. The $\mathrm{Cu}-$ and P-segregated GBs were the same as those used in the previous work $[18,19]$. The initial cell size was $4.05 \times 7.02 \times 14.89 \AA^{3}$. Geometry optimization calculations were performed using the Cambridge Serial Total Energy Package (CASTEP) [25], in which density functional theory $[26,27]$ was used with a plane wave basis set to calculate the electronic properties of solids from first principles. The exchange-correlation interactions were treated using the spin-polarized version of the generalized gradient approximation within the scheme due to Perdew-Burke-Ernzerhof [28]. All calculations were done on the ferromagnetic state. The ultrasoft pseudopotentials [29] represented in reciprocal space were used for all elements in the calculations. The Kohn-Sham wave functions of valence electrons were expanded to the plane wave basis set within a specified cutoff energy (300 eV). The Brillouin zone was sampled using a MonkhorstPack $6 \times 4 \times 2$ k-point mesh and Gaussian smearing with $0.1 \mathrm{eV}$ width.

After the geometry optimization calculation including cell optimization, a uniaxial tensile strain with an increment of $2 \%$ in the [111] direction, which was normal to the GB plane, was applied to the cells for the first-principles fully-relaxed tensile tests. The lattice dimensions in the GB plane were fixed, neglecting Poisson's ratio to simplify the calculation [13-16,18,19]. This step was repeated until GB fracture occurred. In each step, all atomic positions were optimized in accordance with HellmanFeynman forces until all forces were less than $0.03 \mathrm{eV} / \AA$. In the present study, a difference in binding energy between the GB and the free surface was calculated in the static case where the fracture surface was arbitrarily set between the two atomic layers in the GB, and the dynamic case where the fracture surface was the surface after GB fracture by the first-principles fully relaxed tensile test. 


\section{Results}

Figure 2 shows stress-strain curves for the clean GB and the Al-, $\mathrm{Cu}$ - and P-segregated GBs. It is experimentally well known that $\mathrm{P}$ is a strong Fe GB embrittler [30]. Nachtrab et al. [31] experimentally demonstrated that ductility loss in Fe alloys is caused by addition of $\mathrm{Cu}$, which results from both grain boundary precipitation and segregation. Also, it was experimentally shown that $\mathrm{Al}$ induced ductility loss in Fe alloys. Thus, the simulation result that $\mathrm{P}, \mathrm{Cu}$ and $\mathrm{Al}$ are embrittlers of $\mathrm{Fe}$ agrees with the experimental facts. Inspection of Fig. 2 shows that $\mathrm{Al}$ is as strong an embrittler as $\mathrm{Cu}$, although it is a weaker embrittler than P. In general, ductility depends on many microstructural factors such as the concentration of segregation impurities, the grain size and the grain boundary misorientation. Besides, GB precipitation affects GB embrittlement. To the authors' knowledge, there are no experimental data which demonstrate that $\mathrm{Al}$ is as strong an embrittler as $\mathrm{Cu}$. In addition, effects of segregation on the stress-strain behaviour depend on the segregation site [32]. Therefore, the intergranular embrittling potency of $\mathrm{Al}$ is not always the same as that of $\mathrm{Cu}$. Consequently, the simulation result in Fig. 2 suggests that $\mathrm{Al}$ occasionally play a role as a GB embrittler as well as $\mathrm{Cu}$. The effects of an impurity on GB decohesion can be divided into the chemical effect related to the chemical bonding, and the mechanical effect due to a size difference between an impurity atom and a host atom $[7,9,12]$. The atomic size of $\mathrm{Al}$ is larger than that of $\mathrm{Fe}$, while $\mathrm{Cu}$ is almost the same size as Fe. Schweinfest et al. [5] showed that the atomic size difference plays a critical role in the enhanced GB embrittlement of $\mathrm{Cu}$ by $\mathrm{Bi}$ segregation. In addition, Geng et al. [33] pointed out the importance of the volume effect for high accuracy measurement of enhancement of GB embrittlement by segregation. However, it seems that the mechanical effect on the flow stress is small in 
the cases considered in the present work, because of no residual compressive stress prior to straining [15].

According to R-W model [1], the binding energy at the GB, $\Delta E_{G B}$, and at the free surface, $\Delta E_{S F}$, and the binding energy difference, $\Delta E_{G B}-\Delta E_{F S}$, are shown in Table 1 . It can be seen that the intergranular embrittling potency of $\mathrm{Al}$ is much lower than that of $\mathrm{Cu}$ in both the static and the dynamic cases, based on the R-W model. Note that the $\mathrm{Al}$ is a cohesion enhancer in the static case, which agrees with the result by Geng et al. [22]. Thus, there is a large difference between the results from the R-W model and from the first-principles tensile tests, suggesting that variations in the atomic and electronic structures with straining play a vital role in GB embrittlement enhanced by $\mathrm{Al}$ segregation. The difference in binding energy between the static case and the dynamic case is discussed later. The binding energy difference in the P-segregated GB in the present work is larger than that in the previous work [7]. This may be due to difference of $\mathrm{P}$ segregation site: the $\mathrm{P}$ atom is located at the substitutional position [20] in the present work, while it is located at the interstitial position in the previous work [7].

The atomic configuration of $(1 \overline{1} 0)$ in the clean $\mathrm{GB}$ and the $\mathrm{Al}-, \mathrm{Cu}-$ and $\mathrm{P}-$ segregated GBs during straining are shown in Fig. 3. Inspection of Fig. 3 reveals that the first bond breaking occurs at the Fe1-Fe3 bond in the Al-segregated GB. The same trend is found in the $\mathrm{Cu}$-segregated GB, but not in the P-segregated GB.

The numbers of $s, p$ and $d$ electrons in the Fe1 atom, which are obtained by the Mulliken population analysis [34], are listed in Table 2. No charge transfer from the Fe atom to the $\mathrm{Al}$ atom occurs in the $\mathrm{Al}$-segregated GB. This cannot be explained from the electronegativity. Charge transfer weakens the neighboring Fe-Fe bond, resulting in the first bond breaking of the $\mathrm{Fe}-\mathrm{Fe}$ in the $\mathrm{Cu}$-segregated $\mathrm{GB}$ [19]. In the Al-segregated GB, however, charge transfer is not responsible for the first bond breaking at the Fe-Fe bond. 
Figure 4 shows the partial density of states (PDOS) for the Fe1 and the Fe2 atoms in the clean $\mathrm{GB}$, and for the $\mathrm{Fe} 1$ and the $\mathrm{Al}, \mathrm{Cu}$ and $\mathrm{P}$ atoms in the segregated GBs. Also, differences between the PDOS for the Fe1 in the segregated GBs and that in the clean GB are shown in Fig. 5, where the positive value means an increase in PDOS by segregation. It appears from Fig. 5 that the $d$-electrons in the Fe1 atom in the Alsegregated GB are more localized than those in the clean GB, though it is not so much as in the P-segregated GB. In addition, hybridization peaks associated with the $s$ - and $p$ electrons are observed at -7 to $-6 \mathrm{eV}$ in the $\mathrm{Fe} 1$ and the $\mathrm{Al}$ atoms. Such hybridization peaks were found in the Fe1 and the P atoms in P-segregated GB [18]. On the other hand, there were no hybridization peaks in the clean GB and the $\mathrm{Cu}$-segregated GB [19]. Hence, the Fe1-Al bond in the Al-segregated GB has covalent-like characteristics, as does the Fe1-P bond in the P-segregated GB. But, note that the first bond breaking does not occur at the Fe1-Al bond, despite its covalent-like bonding.

\section{Discussion}

The charge density at the bond critical point (BCP) is crucial for bond breaking [35-37]. Hence, the variation in charge density at the $\mathrm{BCP}$ with strain was investigated at the atomic bond where the first breaking occurred, that is, the Fe1-Fe3 bond in the clean GB and the Al- and Cu-segregated GBs, and the Fe1-P bond in the P-segregated GB. The results are shown in Fig. 6, where the charge density variation is the ratio of the charge density at a given strain to that at $0 \%$ strain, and the strain is the strain of the Fe1-Fe3 or Fe1-P bond. In the P-segregated GB, the strain of the Fe1-P bond and the variation in charge density at the $\mathrm{BCP}$ are very low. This is due to the strong covalentlike characteristics of the Fe-P bond. On the other hand, a decrease in charge density with strain is most significant in the $\mathrm{Cu}$-segregated GB. This is because charge transfer 
is enhanced during straining in the $\mathrm{Cu}$-segregated GB [19]. The decrease in charge density is reduced in the Al-segregated GB compared to the other GBs. It appears that a charge density variation of 0.3 is critical for the bond breaking of $\mathrm{Fe} 1-\mathrm{Fe} 3$ in the clean GB and the $\mathrm{Cu}$-segregated GB. However, the critical value for the Fe1-Fe3 bond breaking is larger in the Al-segregated GB.

Figure 7 shows the charge density along the line, normal to the Fe1-Fe3 (or the Fe1-P in the P-segregated GB) bond, passing through the BCP of the Fe1-Fe3 (or the Fe1-P) bond. The charge density at the BCP at $0 \%$ strain in the $\mathrm{Cu}$-segregated GB is larger than that in the clean GB, and the curvature of the former is larger than that of the latter, indicating that the electrons are more localized prior to straining in the $\mathrm{Cu}-$ segregated GB. However, the charge density in the $\mathrm{Cu}$-segregated GB decreases rapidly with the strain, in agreement with the result in Fig. 6, and the curve of charge density at the $\mathrm{BCP}$ becomes flat at $20 \%$ strain which is just before bond breaking. On the other hand, the charge density at the BCP decreases less rapidly in the clean GB than in the $\mathrm{Cu}$-segregated GB, hence the curve of charge density becomes flat at $28 \%$ strain which is just before bond breaking, not at $20 \%$ strain. Breaking of a metallic bond occurs through the transition state where the curvature of the charge density becomes zero [37]. The bond force of $\mathrm{Fe} 1-\mathrm{Fe} 3$ in the $\mathrm{Cu}$-segregated GB is roughly the same as that in the clean GB, but the former decreases more rapidly than the latter. It is suggested, therefore, that the effect of the charge transfer induced by $\mathrm{Cu}$ increases with the strain. In the Al-segregated GB, the charge density curve varies in the same manner as in the clean GB. However, the bond breaking of Fe1-Fe3 in the Al-segregated GB occurs at $22 \%$ strain although the curve of charge density at the BCP is not flat at $20 \%$ strain. As shown in Fig. 7 (d), the charge density at the BCP varies little with the strain in the Psegregated GB. Thus, the bond breaking due to the covalent-like characteristics does not 
occur through the transition state of zero curvature of the charge density. This trend is found in the bond breaking of Fe1-Fe3 in the Al-segregated GB. It should be noted that the bond breaking of $\mathrm{Fe} 1-\mathrm{Fe} 3$ in the Al-segregated GB is partially in the manner of covalent characteristics, despite the bond being between metal atoms.

The PDOS of the Fe1 atom just before and after the bond breaking is shown in Fig. 8. In the clean GB and the $\mathrm{Cu}$-segregated GB, because the PDOS varies with the strain, the PDOS just before bond breaking is different from that prior to straining; however, the PDOS just before bond breaking is almost the same as after bond breaking. These facts agree with the result that bond breaking occurs through the transition state. On the other hand, in the Al- and the P-segregated GBs, the PDOS hardly varies with strain, but varies sharply after bond breaking, suggesting that the mobility of electrons is limited by the covalent-like characteristics before bond breaking, and the electrons are abruptly redistributed to the surface after bond breaking. In general, when an atomic bond is strained, redistribution of electrons enables the bond to deform to the extent that corresponds to the applied strain, resulting in relaxation of the unstable state induced by the strain. The transition state for bond breaking is the redistribution of electrons at the BCP to form the fracture surface [37]. However, the redistribution of electrons is limited in the case of covalent-like bonding, with the result that the unstable state is not relaxed and premature bond breaking occurs even if the charge density at the $\mathrm{BCP}$ is sufficient to sustain the bond. In the Al-segregated GB, the first bond breaking site was the Fe-Fe bond, which is typical of bond breaking due to charge transfer. However, the bond breaking was related to the reduced bond mobility due to the covalent-like characteristics. It is noteworthy that the covalent-like characteristics of $\mathrm{Al}$ affected the mobility of the Fe-Fe metallic bond. As shown in Fig.7, the variation in the charge density curve around the BCP with strain was the same in the Al-segregated GB as in 
the clean GB, at least until $10 \%$ strain. It is therefore suggested that the effect of the covalent-like characteristics of $\mathrm{Al}$ on the $\mathrm{Fe}-\mathrm{Fe}$ bond is small in the initial stage of straining, but the effect of $\mathrm{Al}$ increases as the charge density of the $\mathrm{Fe}-\mathrm{Fe}$ bond decreases with the strain. It is also of interest to note that the first bond breaking does not occur at the Fe-Al bond, despite the covalent-like bond. The cohesive energy of the FeAl compound $(-4.81 \mathrm{eV} /$ atom $)$ is higher than that of the FeP compound $(-5.22$ eV/atom). Hence, it is suggested that the weak covalent-like bond of Fe-Al is responsible for a certain level of mobility of the Fe-Al bond.

In the R-W thermodynamic model and the static case of the first-principles tensile tests, movements of atoms which are located at the cross-section for fracture are all the same. However, in the dynamic case of the first-principles tensile tests, movement of each atom differs from one another, and the most frangible bond is first broken. After the first bond-breaking, redistribution of electrons occurs and the atoms around the bond-breaking site move in order to be in a more stable state. The movements of the atoms affect the second bond-breaking. The same events are caused in one after another during straining, and finally GB fracture occurs. Thus, atomic events of GB fracture are complicated in the dynamic case. When the GB is segregated by other element atoms, the atomic events of GB fracture are much more complicated. The complicated movements can change the configuration of atoms at the surface. Actually, in the present work, the configuration of atoms at the fracture surface in the dynamic case was different from that in the static case for the segregated GBs. This is responsible for disagreement of the binding energy at the surface in the dynamic case with that in the static case. More importantly, when the atomic movement around the bond-breaking site in the dynamic case is inelastic, the process is irreversible and the work required for GB fracture is not entirely converted to the binding energy at the 
surface. Therefore, it is suggested that a difference between the intergranular embrittling potency by the R-W model and that obtained by the first-principles fully relaxed tensile tests in the present work results from irreversible movement of atoms during straining in the first-principles fully relaxed tensile tests.

In the present work, the bond breaking behaviour in the Fe GB is also investigated in terms of variation in electronic structure with straining. In the clean GB and $\mathrm{Cu}$-segregated $\mathrm{GB}$, the redistribution of electrons occurs gradually with increasing strain, which relaxes unstable state induced by the strain. This is the characteristic in the breaking of metallic bonds. In contrast, in the Al- and P-segregated GBs, the redistribution of electrons is limited and the unstable state is not relaxed, resulting in premature bond breaking. This is the characteristic in the breaking of covalent-like bonds. Finally, segregated $\mathrm{Al}$ induces the premature breaking of $\mathrm{Fe}-\mathrm{Fe}$ bond because of not charge transfer but electron mobility limited by the covalent-like characteristics.

\section{Summary}

We have performed first-principles fully relaxed tensile tests on $\Sigma 3(111) /[1 \overline{1} 0]$ tilt Fe GBs segregated with $\mathrm{Al}, \mathrm{Cu}$ or $\mathrm{P}$. The calculations suggested that the intergranular embrittling potency of $\mathrm{Al}$ was as strong as that of $\mathrm{Cu}$. On the other hand, according to the $\mathrm{R}-\mathrm{W}$ model, the intergranular embrittling potency of $\mathrm{Al}$ was much lower than that of $\mathrm{Cu}$. Thus, there was a large difference in estimation of the embrittling potency of $\mathrm{Al}$ between the R-W model and the first-principles tensile tests.

The first bond breaking occurred at the $\mathrm{Fe}-\mathrm{Fe}$ bond neighbouring an $\mathrm{Al}$ atom in the Al-segregated GB, which is typical of bond breaking due to charge transfer. However, no charge transfer was observed from the neighbouring Fe atom to the $\mathrm{Al}$ atom, while the neighbouring Fe showed covalent-like characteristics. The Fe-Fe 
metallic bond had covalent-like characteristics imparted by segregated Al, resulting in premature breaking of the Fe-Fe bond. Investigations of the charge density at the bond critical point suggested that the effect of covalent-like characteristics of $\mathrm{Al}$ on the $\mathrm{Fe}-\mathrm{Fe}$ bond was small in the initial stage of straining, but it increased as the charge density of the Fe-Fe bond decreased with increasing strain.

There was a difference in binding energy between the static case and the dynamic case. The binding energy at the surface in the dynamic case was lower than that in the static case, because atoms moved during straining in order to be in a more stable state. If atoms move significantly during straining and the atomic configuration at the facture surface for the dynamic case is remarkably different from that for the static case, the first-principles fully relaxed tensile tests are required to accurately investigate effects of segregation on GB decohesion.

\section{Acknowledgements}

This work was supported by a Grant-in-Aid for JSPS Fellows (22.5568). Computation time was provided by the SuperComputer Laboratory, Institute for Chemical Research, Kyoto University. 


\section{References}

[1] J. R. Rice and J. S. Wang, Mater. Sci. Eng. A 10723 (1989).

[2] C. L. Briant and S. K. Banerji, Int. Met. Rev. 23164 (1978).

[3] W. Losch, Acta Metall. 271885 (1979).

[4]R. P. Messmer andC. L. Briant, Acta Metall. 30457 (1982).

[5] R. Schweinfest, A. T. Paxton, and M. W. Finnis, Nature 431008 (2004).

[6] M. Yamaguchi, M. Shiga, and H. Kaburaki Science 307393 (2005).

[7] R. Wu, A. J. Freeman, and G. B. Olson, Science 265376 (1994).

[8] R. Wu, A. J. Freeman, and G. B. Olson, Phys. Rev. B 537504 (1996).

[9] W. T. Geng, A. J. Freeman, R. Wu, G. B. Geller, and J. E. Raynolds, Phys. Rev. B 607149 (1999).

[10] L. Zhong, R. Wu, A. J. Freeman, and G. B. Olson, Phys. Rev. B 6213938 (2000).

[11] J. S. Braithwaite and P. Rez, Acta Mater. 532715 (2005).

[12] J. X. Shang, X. D. Zhao, F. H. Wang, C. Y. Wang, and H. B. Xu, Comput. Mater. Sci. 38217 (2006).

[13] G. H. Lu, S. Deng, T. Wang, M. Kohyama, and R. Yamamoto, Phys. Rev. B 69 134106 (2004).

[14] G. H. Lu, Y. Zhang, S. Deng, T. Wang, M. Kohyama, R. Yamamoto, F. Liu, K. Horikawa, and M. Kanno, Phys. Rev. B 73224115 (2006).

[15] Y. Zhang, G. H. Lu, T. Wang, S. Deng, M. Kohyama, and R. Yamamoto, Mater. Trans. 472678 (2006).

[16] Y. Zhang, G. H. Lu, S. Deng, T. Wang, H. Xu, M. Kohyama and R. Yamamoto, Phys. Rev. B 75174101 (2007).

[17] H. Z. Zhang, L. Liu, and S. Q. Wang, Comput. Mater. Sci. 38800 (2007).

[18] M. Yuasa and M. Mabuchi, Phys. Rev. B 82094108 (2010).

[19] M. Yuasa and M. Mabuchi, J. Phys.: Condens. Matter 22505705 (2010).

[20] M. Yamaguchi, Metall. Mater. Trans. A 42319 (2011).

[21]M. P. Seah, Acta Metall. 28955 (1980).

[22] W. T. Geng, A. J. Freeman, and G. B. Olson, Phys. Rev. B 63165415 (2001).

[23] B. Mintz and J. M. Arrowsmith, Metals Technol. 624 (1979).

[24] T. Uesugi, M. Kohyama, and K. Higashi Phys. Rev. B 6818410 (2003).

[25] M. C. Payne, M. P. Teter, D. C. Allan, T. A. Arias, and J. D. Joannopoulos, Rev. Mod. Phys. 641045 (1992). 
[26] P. Hohenberg and W. Kohn, Phys. Rev. 136 B864 (1964).

[27] W. Kohn and L. Sham, Phys. Rev. 140 A1133 (1965).

[28] J. P. Perdew, K. Burke, and M. Ernzerhof, Phys. Rev. Lett. 773865 (1996).

[29] D. Vanderbilt, Phys. Rev. B 417892 (1990).

[30]S. Takayama, T. Ogura, S. C. Fu, and C. J. Macmahon, Jr., Metall.Trans. A 11A 1513 (1980).

[31] W. T. Nachtrab and Y. U. Chou, Metall. Trans. A 17A 1995 (1986).

[32] M. Yuasa and M. Mabuchi, Mater. Trans. 521369 (2011).

[33] W. T. Geng, A. J. Freeman, R. Wu and G. B. Olson, Phys. Rev. B 626208 (2000).

[34] R. S. Mulliken, J. Chem. Phys. 231833 (1955).

[35] M. E. Eberhart, Acta Mater. 442495 (1996).

[36] N. Kioussis, M. Herbranson, W. Collins, and M. E. Eberhart, Phys. Rev. Lett. 88 125501 (2002).

[37] M. E. Eberhart, D. P. Clougherty, and J. M. MacLaren, J. Am. Chem. Soc. 115 5262 (1993). 


\section{Table}

Table 1 The binding energy (in $\mathrm{eV}$ /atom) at the grain boundaries, $\Delta E_{G B}$, and at the free surface, $\Delta E_{S F}$, and the binding energy difference, $\Delta E_{G B}-\Delta E_{S F}$, at the Al-, $\mathrm{Cu}-$ and $\mathrm{P}-$ segregated grain boundaries. Two cases are calculated: the static case where the fracture surface is arbitrarily set between the two atomic layers in the grain boundary, and the dynamic case where the fracture surface is the surface after grain boundary fracture by the first-principles fully relaxed tensile test.

\begin{tabular}{lcccc}
\hline & & $\Delta E_{G B}$ & $\Delta E_{F S}$ & $\Delta E_{G B}-\Delta E_{F S}$ \\
\hline Al-segregated GB & Static & -4.99 & -4.97 & -0.01 \\
& Dynamic & -4.99 & -5.07 & 0.08 \\
Cu-segregated GB & Static & -3.42 & -3.98 & 0.56 \\
& Dynamic & -3.42 & -4.17 & 0.75 \\
P-segregated GB & Static & -4.80 & -6.42 & 1.63 \\
& Dynamic & -4.80 & -6.78 & 1.99 \\
\hline
\end{tabular}


Table 2 The numbers of $s, p$ and $d$ electrons in the Fe1 atom at the clean grain boundary and the Al-, $\mathrm{Cu}$ - and $\mathrm{P}$-segregated grain boundaries. The numbers of electrons are obtained by the Mulliken population analysis. No charge transfer from the $\mathrm{Fe} 1$ atom to the $\mathrm{Al}$ atom occurs at the Al-segregated grain boundary.

\begin{tabular}{lrrrr}
\hline & $3 d$ & $4 s$ & $4 p$ & Total \\
\hline clean GB & 6.68 & 0.71 & 0.64 & 8.03 \\
Al-segregated GB & 6.73 & 0.69 & 0.65 & 8.07 \\
Cu-segregated GB & 6.68 & 0.68 & 0.55 & 7.90 \\
P-segregated GB & 6.75 & 0.68 & 0.60 & 8.02 \\
\hline
\end{tabular}




\section{Figure captions}

Figure 1 Unit cell model of Fe with a $\Sigma 3(111) /[1 \overline{1} 0]$ tilt grain boundary. Four cells are used: one is the cell without segregation at the grain boundary (the clean GB); the others are the cells with $\mathrm{Al}, \mathrm{Cu}$ or $\mathrm{P}$ segregation at the grain boundary (the $\mathrm{Al}-, \mathrm{Cu}-$ or $\mathrm{P}$ segregated $\mathrm{GBs}$ ), in which an $\mathrm{Fe}$ atom $(\mathrm{Fe} 2)$ at the grain boundary is substituted by the segregation atom. The initial cell size is $4.05 \times 7.02 \times 14.89 \AA^{3}$. The black and gray circles indicate $\mathrm{Fe}$ atoms on the $(1 \overline{1} 0)$ and $(2 \overline{2} 0)$, respectively.

Figure 2 Stress-strain curves for the clean grain boundary and the Al-, $\mathrm{Cu}-$ and $\mathrm{P}-$ segregated grain boundaries (after Refs [18,19]).

Figure 3 Atomic configuration of (1 $\overline{1} 0)$ : (a) $20 \%$ strain in the Al-segregated grain boundary, (b) $22 \%$ strain in the Al-segregated grain boundary, (c) $20 \%$ strain in the $\mathrm{Cu}$ segregated grain boundary, (d) $22 \%$ strain in the $\mathrm{Cu}$-segregated grain boundary, (e) $10 \%$ strain in the P-segregated grain boundary, (f) $12 \%$ strain in the P-segregated grain boundary (after Ref. [18]). The first bond breaking occurs at the Fe1-Fe3 bond in the $\mathrm{Al}-$ and $\mathrm{Cu}$-segregated grain boundaries.

Figure 4 Partial density of states (PDOS) of the $\mathrm{Fe} 1, \mathrm{Fe} 2, \mathrm{Al}, \mathrm{Cu}$ and $\mathrm{P}$ atoms at $0 \%$ strain: (a) Fe1 in the clean grain boundary, (b) Fe1 in the Al-segregated grain boundary, (c) $\mathrm{Fe} 1$ in the $\mathrm{Cu}$-segregated grain boundary, (d) Fe1 in the P-segregated grain boundary, (e) $\mathrm{Fe} 2$ in the clean grain boundary, (f) $\mathrm{Al}$ in the Al-segregated grain boundary, $(\mathrm{g}) \mathrm{Cu}$ in the $\mathrm{Cu}$-segregated grain boundary, and (h) $\mathrm{P}$ in the $\mathrm{P}$-segregated grain boundary. The Fermi level defines the zero of energy. Magnified figures of the PDOS in the range -13 to $-5 \mathrm{eV}$ are shown on the upper left. The arrow indicates the hybridization peak between the $\mathrm{Fe} 1$ and the $\mathrm{Al}$ atoms in the Al-segregated GB.

Figure 5 Differences between the partial density of states (PDOS) for the Fe1 in the segregated GBs and that in the clean GB:(a) s-electrons, (b) p-electrons and (c) delectrons. The positive value means an increase in PDOS by segregation. The Fermi level defines the zero of energy. The arrow indicates the hybridization peak between the $\mathrm{Fe} 1$ and the $\mathrm{Al}$ atoms in the Al-segregated GB.

Figure 6 Variations in charge density at the bond critical point of the atomic bond for the first bond breaking (the $\mathrm{Fe} 1-\mathrm{Fe} 3$ bond in the clean grain boundary and the Al- and $\mathrm{Cu}$-segregated grain boundaries, and the Fe1-P bond in the P-segregated grain boundary) with strain. The charge density variation is the ratio of the charge density at a given strain to that at $0 \%$ strain, and the strain is the strain of the Fe1-Fe3 or Fe1-P bond.

Figure 7 Charge density along the line, normal to the Fe1-Fe3 bond (or the Fe1-P bond in the P-segregated grain boundary), passing through the bond critical point of the Fe1Fe3 bond: (a) the clean grain boundary, (b) the Al-segregated grain boundary, (c) the $\mathrm{Cu}$-segregated grain boundary, and (d) the P-segregated grain boundary. The curve of charge density becomes flat at the strain at which bond breaking occurs in the clean grain boundary and the $\mathrm{Cu}$-segregated grain boundary, while the transition state of flat charge density is not found in the Al- and P-segregated grain boundaries.

Figure 8 Partial density of states (PDOS) of the Fe1 atom just before bond breaking. PDOS at (a) $28 \%$ in the clean grain boundary, (b) $20 \%$ in the Al-segregated grain 
boundary, (c) $20 \%$ in the Cu-segregated grain boundary and (d) $10 \%$ in the P-segregated grain boundary (after Ref. [18]), and after the bond breaking; (e) 30\% in the clean grain boundary, (f) $22 \%$ in the Al-segregated grain boundary, (g) $22 \%$ in the $\mathrm{Cu}$-segregated grain boundary, and (h) $12 \%$ in the P-segregated grain boundary. The Fermi level defines the zero of energy. 
Table 1 The segregation energy (in $\mathrm{eV} /$ atom) at the grain boundaries, $\Delta E_{G B}$, and at the free surface, $\Delta E_{S F}$, and the segregation energy difference, $\Delta E_{G B}-\Delta E_{S F}$, at the Al-, $\mathrm{Cu}$ - and $\mathrm{P}$ segregated grain boundaries. Two cases are calculated: the static case where the fracture surface is arbitrarily set between the two atomic layers in the grain boundary, and the dynamic case where the fracture surface is the surface after grain boundary fracture by the first-principles fully relaxed tensile test.

\begin{tabular}{ccccc}
\hline & & $\Delta E_{G B}$ & $\Delta E_{F S}$ & $\Delta E_{G B}-\Delta E_{F S}$ \\
\hline Al-segregated GB & Static & -4.99 & -4.97 & -0.01 \\
& Dynamic & -4.99 & -5.07 & 0.08 \\
Cu-segregated GB & Static & -3.42 & -3.98 & 0.56 \\
& Dynamic & -3.42 & -4.17 & 0.75 \\
P-segregated GB & Static & -4.80 & -6.42 & 1.63 \\
& Dynamic & -4.80 & -6.78 & 1.99 \\
\hline
\end{tabular}


Table 2 The numbers of $s, p$ and $d$ electrons in the Fe1 atom at the clean grain boundary and the Al-, $\mathrm{Cu}$ - and P-segregated grain boundaries. The numbers of electrons are obtained by Mulliken population analysis. No charge transfer from the Fe1 atom to the $\mathrm{Al}$ atom occurs at the Al-segregated grain boundary.

\begin{tabular}{lrrrr}
\hline & $3 d$ & $4 s$ & $4 p$ & Total \\
\hline clean GB & 6.68 & 0.71 & 0.64 & 8.03 \\
Al-segregated GB & 6.73 & 0.69 & 0.65 & 8.07 \\
Cu-segregated GB & 6.68 & 0.68 & 0.55 & 7.90 \\
P-segregated GB & 6.75 & 0.68 & 0.60 & 8.02 \\
\hline
\end{tabular}




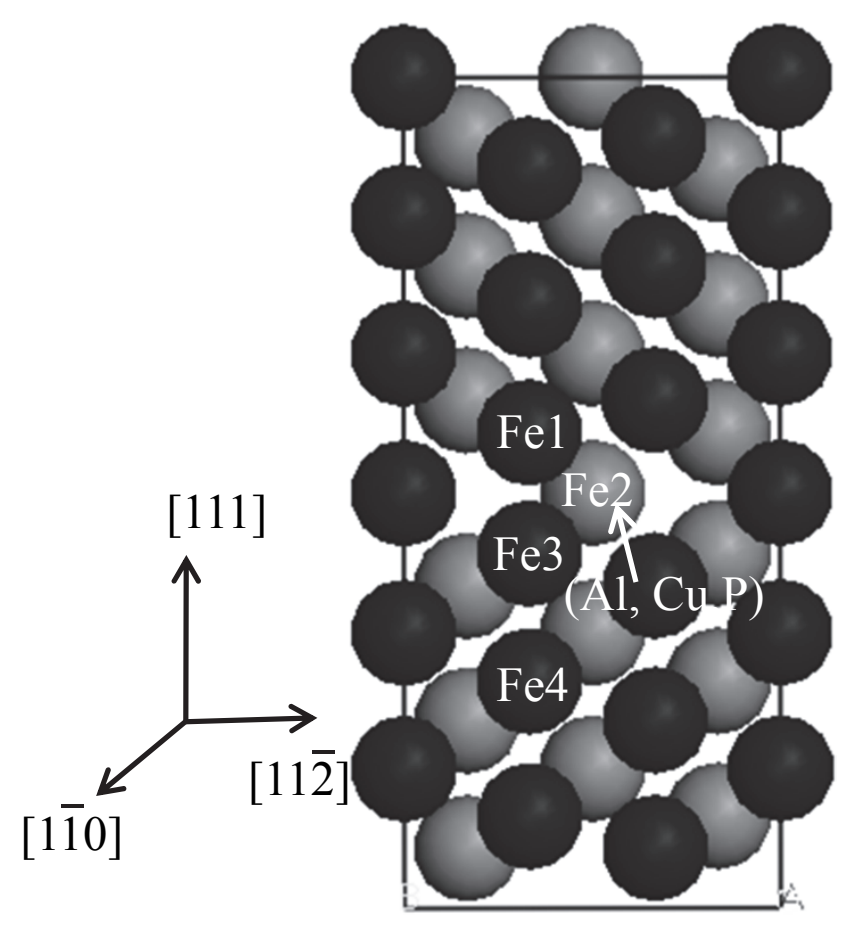

Fig. 1 Unit cell model of Fe with a $\Sigma 3(111) /\left[11^{-}\right]$tilt grain boundary. Four cells are used: one is the cell without segregation at the grain boundary (the clean GB); the others are the cells with $\mathrm{Al}, \mathrm{Cu}$ or $\mathrm{P}$ segregation at the grain boundary (the $\mathrm{Al}-\mathrm{Cu}$ - or P-segregated GBs), in which an Fe atom (Fe2) at the grain boundary is substituted by the segregation atom. The initial cell size is $4.05 \times 7.02 \times 14.89 \AA$. The black and gray circles indicate Fe atoms on (110) and (220), respectively. 


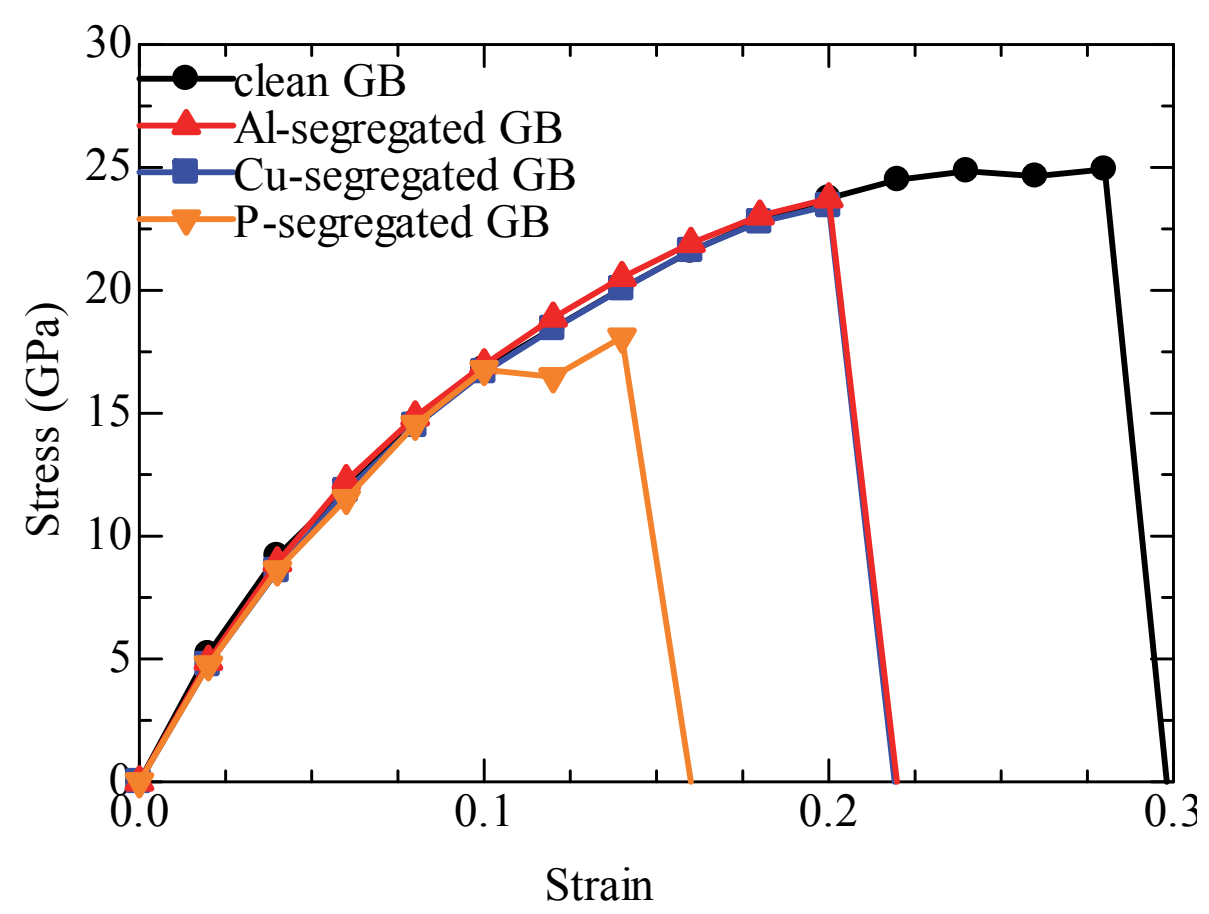

Fig.2 Stress-strain curves for the clean grain boundary and the Al-, $\mathrm{Cu}-$ and $\mathrm{P}$-segregated grain boundaries. $\mathrm{Al}$ is as strong an embrittler as $\mathrm{Cu}$. 

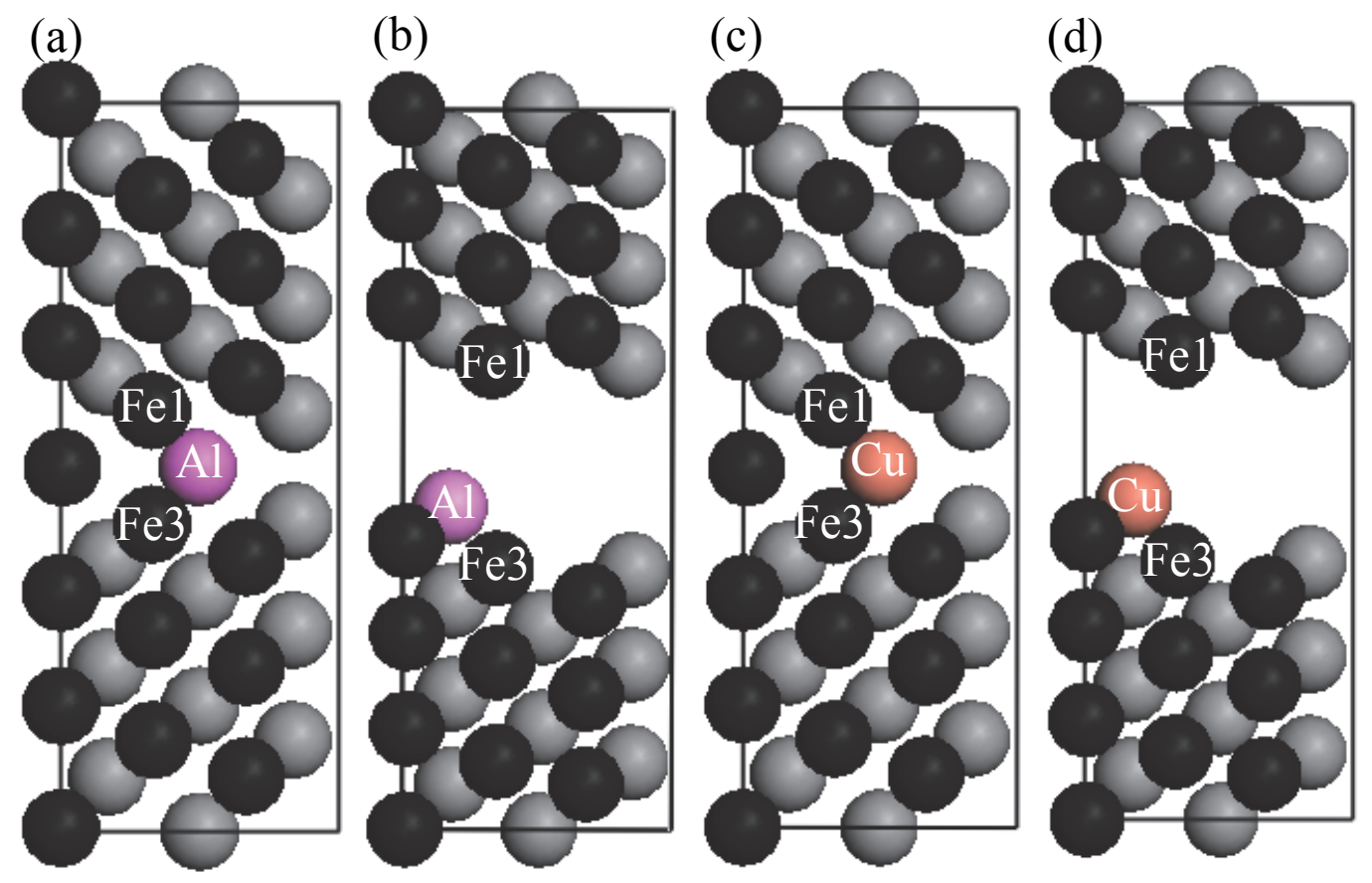

(e)

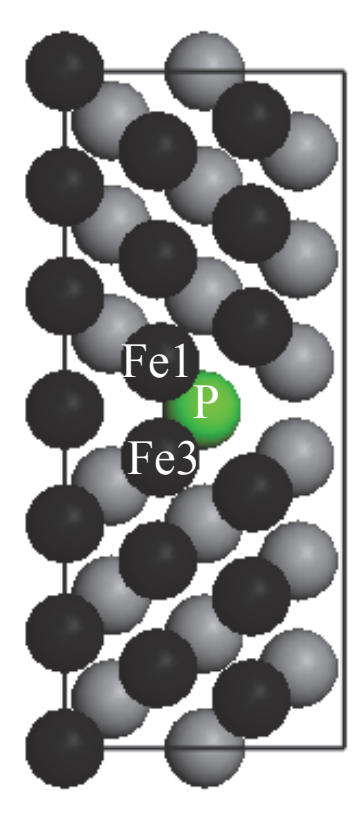

(f)

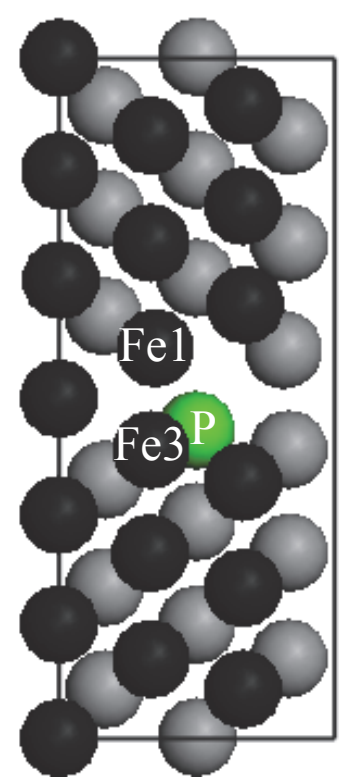

Fig.3 Atomic configuration of (110): (a) 20\% strain in the Al-segregated grain boundary, (b) $22 \%$ strain in the Al-segregated grain boundary, (c) $20 \%$ strain in the $\mathrm{Cu}$-segregated grain boundary, (d) $22 \%$ strain in the Cu-segregated grain boundary, (e) $10 \%$ strain in the Psegregated grain boundary, (f) $12 \%$ strain in the P-segregated grain. The first bond breaking occurs at the $\mathrm{Fe} 1-\mathrm{Fe} 3$ bond in the $\mathrm{Al}$ - and $\mathrm{Cu}$-segregated grain boundaries. 


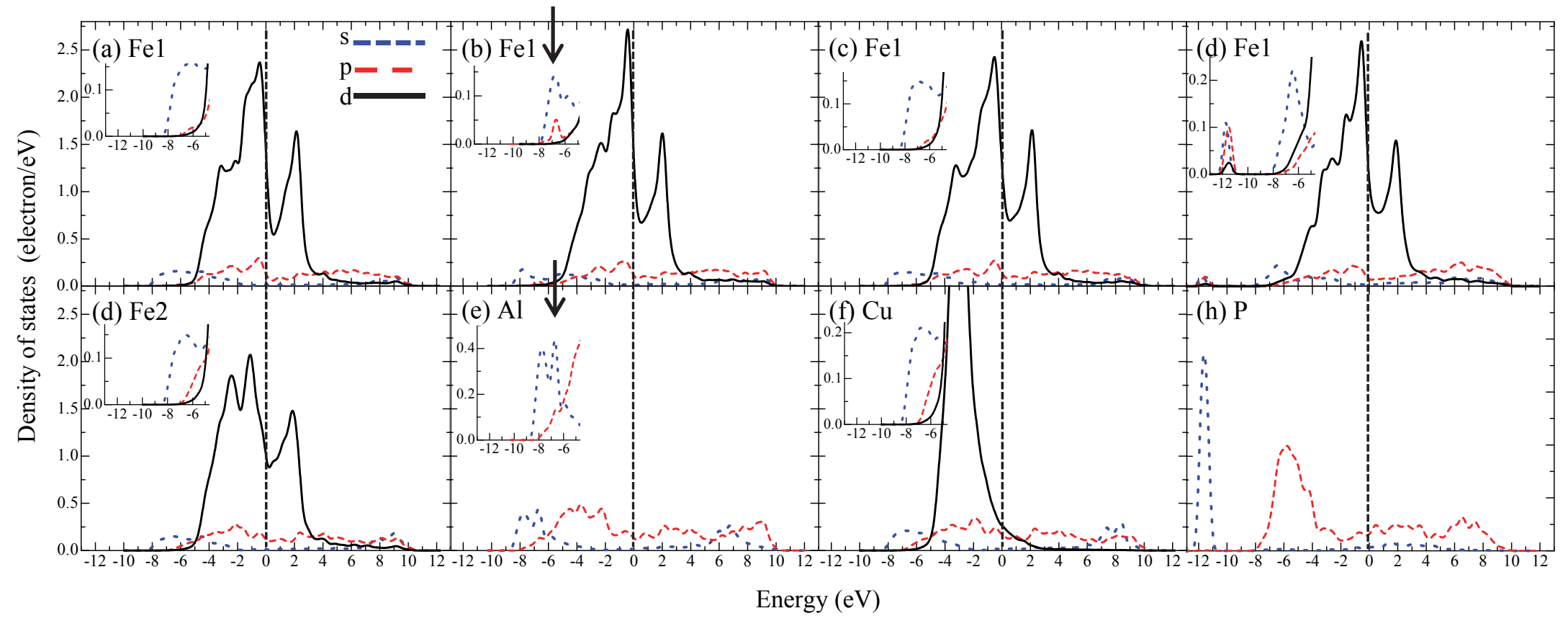

Fig.4 Partial density of states (PDOS) of the Fe1, Fe2, $\mathrm{Al}, \mathrm{Cu}$ and $\mathrm{P}$ atoms at $0 \%$ strain: (a) $\mathrm{Fe} 1$ in the clean grain boundary, (b) $\mathrm{Fe} 1$ in the Al-segregated grain boundary, (c) $\mathrm{Fe} 1$ in the $\mathrm{Cu}$-segregated grain boundary, (d) Fe1 in the P-segregated grain boundary, (e) Fe2 in the clean grain boundary, (f) $\mathrm{Al}$ in the $\mathrm{Al}$-segregated grain boundary, $(\mathrm{g}) \mathrm{Cu}$ in the $\mathrm{Cu}$ segregated grain boundary, and (h) P in the P-segregated grain boundary. The Fermi level defines the zero of energy. Magnified figures of the PDOS in the range -13 to $-5 \mathrm{eV}$ are shown on the upper left. The arrow indicates the hybridization peak between $\mathrm{Fe} 1$ and $\mathrm{Al}$ atoms in the Al-segregated GB. 


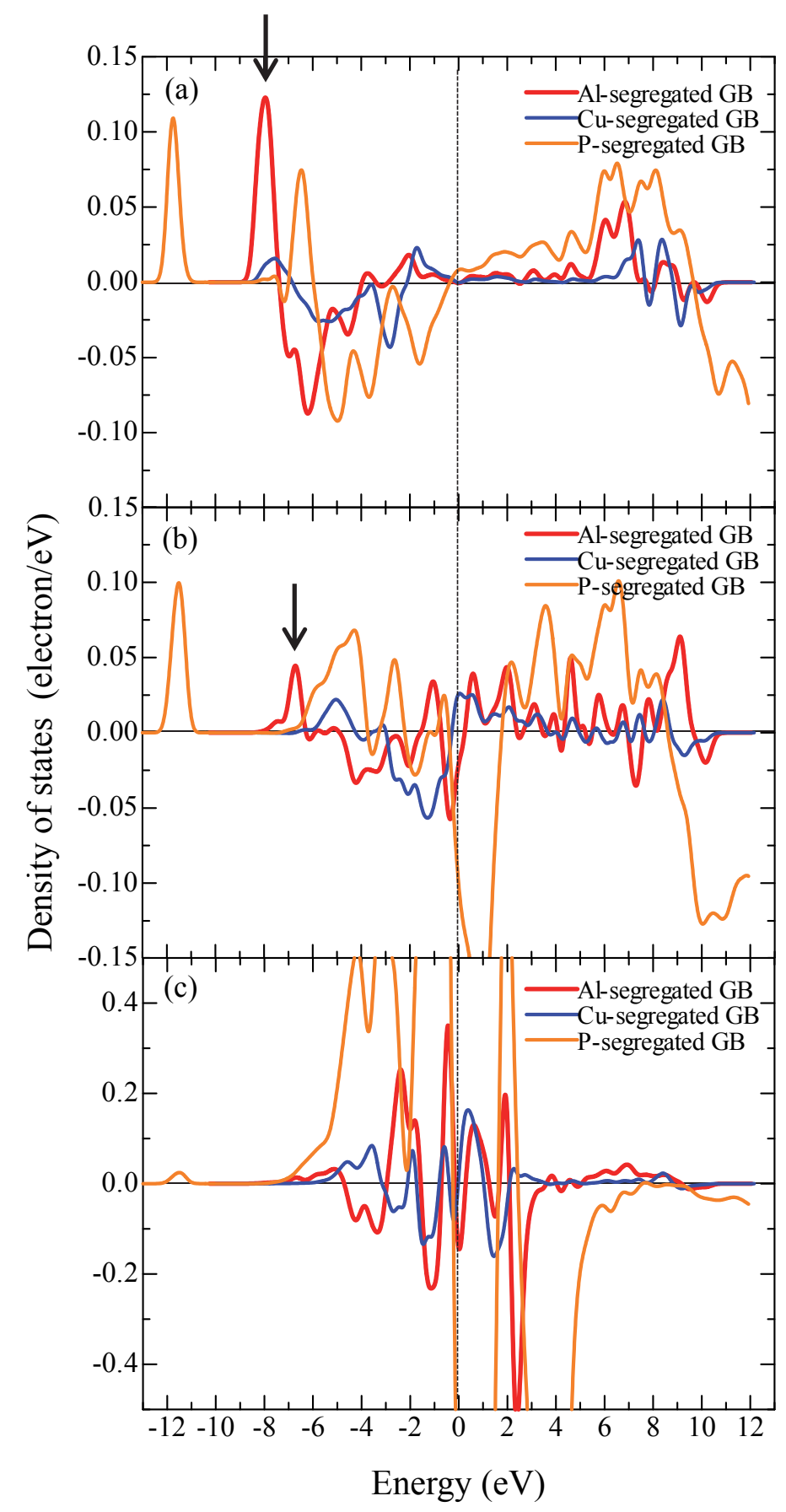

Fig.5 The difference between the Partial density of states (PDOS) for the Fe1 in the segregated GBs and that in the clean GB:(a) s-electrons, (b) p-electrons, and (c) delectrons. The positive value means an increase of PDOS in the segregated GBs. The Fermi level defines the zero of energy. The arrow indicates the hybridization peak between $\mathrm{Fe} 1$ and $\mathrm{Al}$ atoms in the Alsegregated GB. 


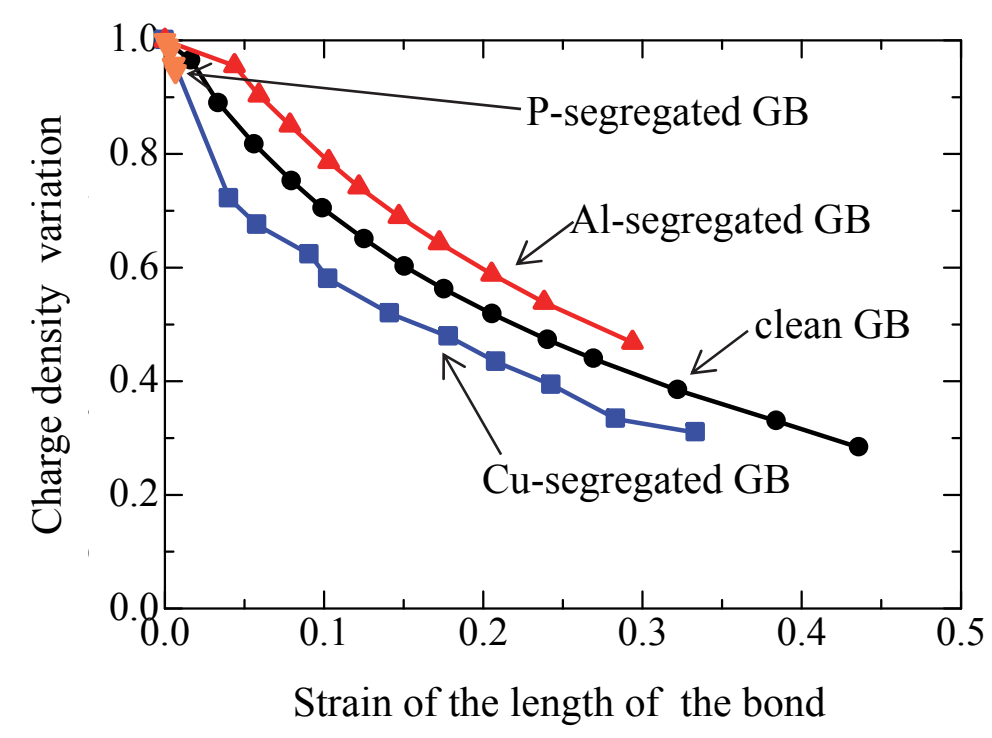

Fig. 6 Variations in charge density at the bond critical point of the atomic bond for the first bond breaking (the $\mathrm{Fe} 1-\mathrm{Fe} 3$ bond in the clean grain boundary and the $\mathrm{Al}$ - and $\mathrm{Cu}$-segregated grain boundaries, and the Fe1-P bond in the P-segregated grain boundary) with strain. The charge density variation is the ratio of the charge density at a given strain to that at $0 \%$ strain, and the strain is the strain of the Fe1-Fe3 or Fe1-P bond. 


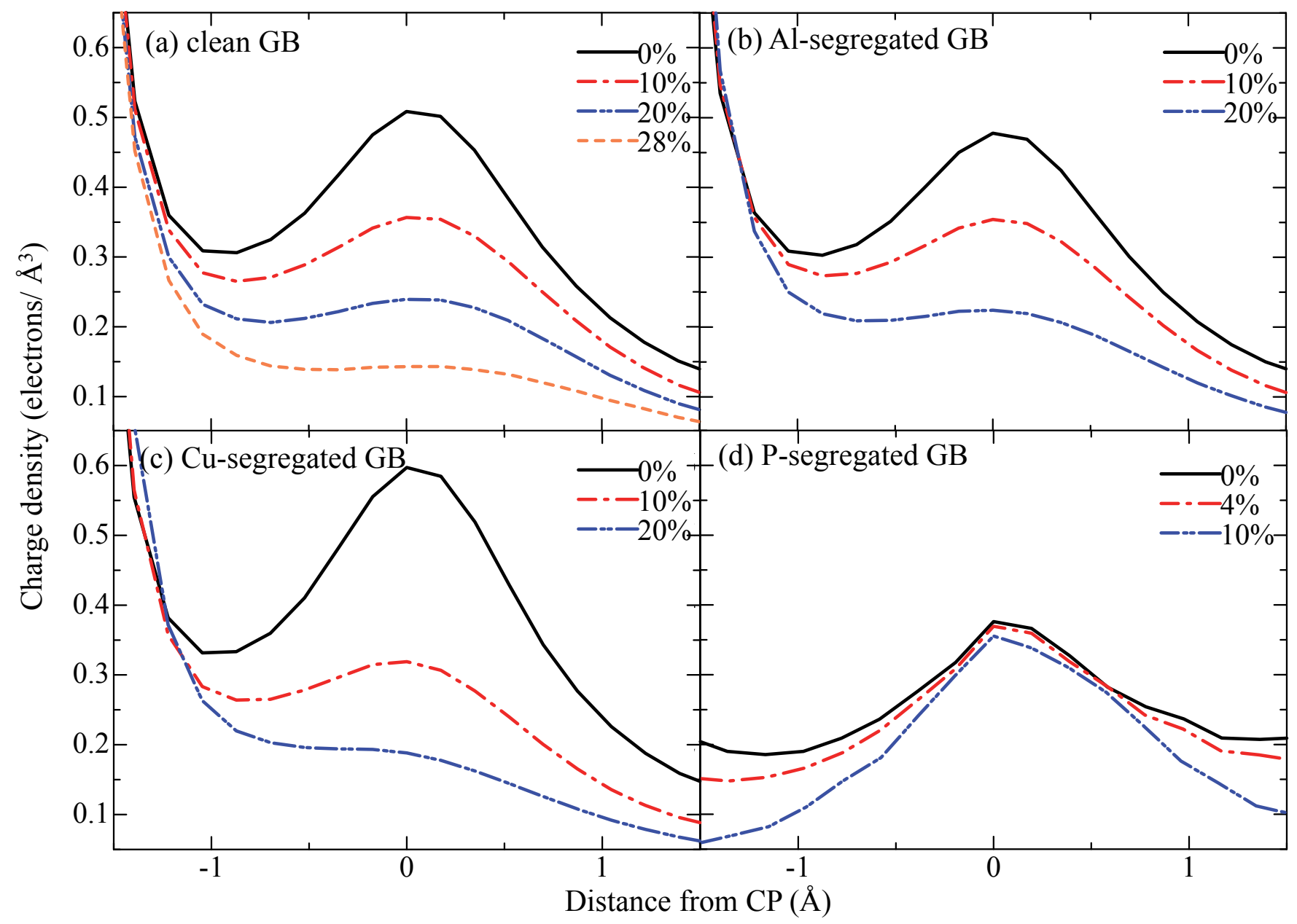

Fig. 7 Charge density along the line, normal to the Fe1-Fe3 bond (or the Fe1-P bond in the Psegregated grain boundary), passing through the bond critical point of the Fe1-Fe3 bond: (a) the clean grain boundary, (b) the Al-segregated grain boundary, (c) the $\mathrm{Cu}$-segregated grain boundary, and (d) the P-segregated grain boundary. The curve of charge density becomes flat at the strain at which bond breaking occurs in the clean grain boundary and the $\mathrm{Cu}$-segregated grain boundary, while the transition state of flat charge density is not found in the Al- and P-segregated grain boundaries. 


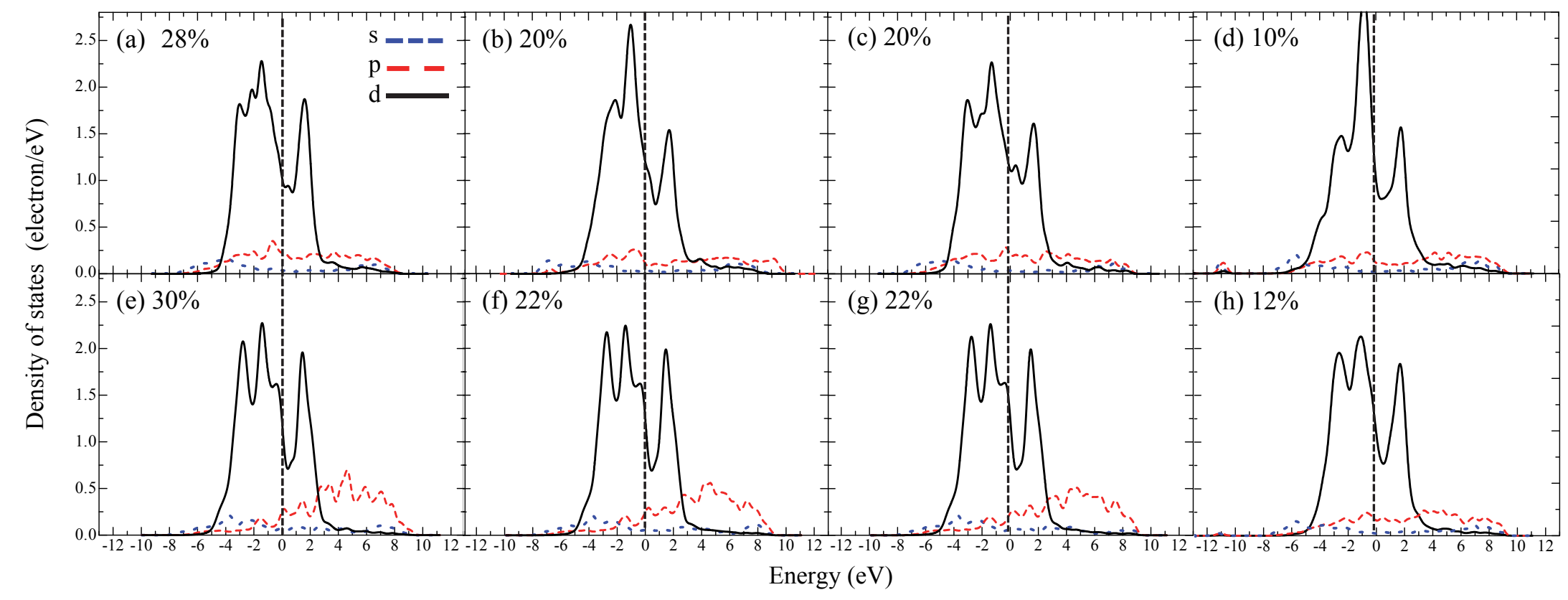

Fig. 8 Partial density of states (PDOS) of the Fe1 atom just before bond breaking. PDOS at (a) $28 \%$ in the clean grain boundary, (b) $20 \%$ in the Al-segregated grain boundary, (c) $20 \%$ in the $\mathrm{Cu}$-segregated grain boundary and (d) $10 \%$ in the P-segregated grain boundary (after Ref. [18]), and after the bond breaking; (e) $30 \%$ in the clean grain boundary, (f) $22 \%$ in the Al-segregated grain boundary, (g) $22 \%$ in the Cu-segregated grain boundary, and (h) $12 \%$ in the P-segregated grain boundary. The Fermi level defines the zero of energy. 